\title{
Accuracy of Thresholds Based on Cadence and Lifestyle Counts per Minute to Detect Outdoor Walking in Older Adults With Mobility Limitations
}

\author{
Sandra C. Webber, Francine Hahn, Lisa M. Lix, Brenda J. Tittlemier, Nancy M. Salbach, \\ and Ruth Barclay
}

\begin{abstract}
Objective: To determine the optimal threshold, based on cadence and lifestyle counts per minute, to detect outdoor walking in mobility-limited older adults. Methods: Older adults $(N=25$, median age: 77.0 years, interquartile range: 10.5$)$ wore activity monitors during 80 outdoor walks. Walking bouts were identified manually (reference standard) and compared with identification using cadence thresholds ( $\geq 30, \geq 35, \geq 40, \geq 45$, and $\geq 50$ steps $/ \mathrm{min})$ and $>760$ counts per minute using low frequency extension analysis. Results: Median walking bout duration was $10.5 \mathrm{~min}$ (interquartile range 4.8) and median outdoor walking speed was $0.70 \mathrm{~m} / \mathrm{s}$ (interquartile range 0.20$)$. Cadence thresholds of $\geq 30, \geq 35$, and $\geq 40 \mathrm{steps} / \mathrm{min}$ demonstrated high sensitivity $(1.0,95 \%$ confidence intervals $[0.95,1.0])$ to detect walking bouts; estimates for specificity and positive predictive value were highest for $\geq 40 \mathrm{steps} / \mathrm{min}$. Conclusion: A cadence threshold of $\geq 40 \mathrm{steps} / \mathrm{min}$ is recommended for detecting sustained outdoor walking in this population.
\end{abstract}

Keywords: accelerometer, low frequency extension, sensitivity, specificity

Mobility in older adults is important for maintaining independence and participation in daily activities. It is often evaluated in clinical and research environments using measures of capacity such as the $10-\mathrm{m}$ walk test $(10 \mathrm{mWT}$; Rydwik, Bergland, Forsen, \& Frandin, 2012) and the 6-min walk test (6MWT; Harada, Chiu, \& Stewart, 1999), but results of these tests are not necessarily related to performance in everyday life (Giannouli, Bock, Mellone, \& Zijlstra, 2016). Because walking is the most common form of physical activity in older adults (Valenti, Bonomi, \& Westerterp, 2016), it is important to accurately measure ambulation in daily life in order to characterize mobility behavior.

Activity monitors that include accelerometers are frequently used to quantify physical activity and walking (Schrack et al., 2016). Often, physical activity is described by the amount of time spent in sedentary, light, and moderate to vigorous intensity aerobic activity based on counts per minute (cpm) thresholds (ActiGraph, 2008; Tryon \& Williams, 1996). Thresholds of $>760 \mathrm{cpm}$ (Gennuso, Gangnon, Matthews, Thraen-Borowski, \& Colbert, 2013; Matthew, 2005) or $>809$ cpm (Hall, Howe, Rana, Martin, \& Morey, 2013) have been suggested to represent moderate activity in older adults.

Other researchers have used step data to specifically describe walking, regardless of activity intensity, in adults during free-living behavior (Granat, Clarke, Holdsworth, Stansfield, \& Dall, 2015; Orendurff, Schoen, Bernatz, Segal, \& Klute, 2008). These studies have shown that most episodes of walking last $<30-60 \mathrm{~s}$ and involve $<40$ continuous steps. When longer, continuous walking events occur, they tend to be associated with higher cadence (steps per minute) values (Granat et al., 2015; Tudor-Locke \& Rowe, 2012).

Webber, Hahn, Tittlemier, and Barclay are with the Department of Physical Therapy, College of Rehabilitation Sciences, University of Manitoba, Winnipeg, MB, Canada. Lix is with the Department of Community Health Sciences, Max Rady College of Medicine, University of Manitoba, Winnipeg, MB, Canada. Salbach is with the Department of Physical Therapy, University of Toronto, Toronto, ON, Canada. Webber (sandra.webber@umanitoba.ca) is corresponding author.
Nevertheless, few studies have attempted to identify episodes of sustained walking in free-living data collected in older adults, and there is no consensus on appropriate methods for analyzing such data.

Tudor-Locke, Camhi, et al. (2011) and Tudor-Locke and Rowe (2012) suggested that cadences between 40 and 59 represent purposeful walking and cadences between 60 and 79 indicate slow walking in adults and older adults. Gardner, Montgomery, Scott, Afaq, and Blevins (2007) collected accelerometer data over 7 days and found that the average cadence of the best $5 \mathrm{~min}$ (i.e., the maximum number of steps taken over a sliding window of $5 \mathrm{~min}$ ) was $68 \mathrm{steps} / \mathrm{min}$ in their sample of 129 healthy older adults (mean 64 years of age, mean 6MWT distance $416 \mathrm{~m}$ ). Cadences approximating $100 \mathrm{steps} / \mathrm{min}$ are achieved during free-living walking in healthy older adults (Paraschiv-Ionescu et al., 2018; Tudor-Locke, Barreira, Brouillette, Foil, \& Keller, 2013; Urbanek et al., 2018), but these levels are rarely sustained (Tudor-Locke et al., 2013). Maximum sustained cadences achieved by people with mobility limitations have not been examined.

The purpose of this evaluation was to determine the optimal threshold for identifying outdoor walking bouts in mobility-limited older adults. Five cadence thresholds $(\geq 30, \geq 35, \geq 40, \geq 45$, and $\geq 50 \mathrm{steps} / \mathrm{min})$ and a lifestyle $\mathrm{cpm}$ threshold $(>760 \mathrm{cpm})$ were evaluated. Identification of the optimal method of detecting walking bouts may have practical implications for detecting walking in freeliving accelerometer data collected in similar samples of older adults.

\section{Methods}

\section{Protocol}

This analysis was conducted as part of a larger trial for communitydwelling older adults with self-reported outdoor mobility limitations. The protocol for the larger randomized controlled trial (Salbach et al., 2019) and our pilot work (Barclay et al., 2018) 
have been published. Participants in the study self-identified as having difficulty walking outdoors because of physical impairments, low confidence, and/or low motivation. Older adults were ineligible for the trial if they engaged in $\geq 150$ min of physical activity per week. In brief, the purpose of the larger study was to determine if there was a difference in outdoor walking activity in older adults assigned to a 1-day educational workshop and a supervised outdoor walking program compared with those assigned to the 1-day educational workshop and weekly walking reminders. Baseline measurements included basic demographics and capacity-based measures such as the 10mWT and the 6MWT (and others detailed in our protocol [Salbach et al., 2019]). For the 10mWT, participants were asked to walk at a comfortable pace and were measured over the middle $10 \mathrm{~m}$ of a 14-m course. Instructions provided for the 6MWT were to walk as far as possible in $6 \mathrm{~min}$. Physical activity was measured for 8 days using ActiGraph GT3X+ activity monitors (ActiGraph LLC, Pensacola, FL) and Qstarz BT-Q1000XT A-GPS travel recorder global positioning system (GPS) devices (Qstarz International Co., Ltd., Taipei, Taiwan). Ethical approval for this study was obtained from research ethics boards at the University of Toronto, University of Manitoba, University of Alberta, and McGill University. All participants provided informed written consent prior to data collection.

This analysis is based on data from 25 older adults who took part in the supervised outdoor walking program in the summer and fall of 2018. The walking program consisted of supervised walking sessions in outdoor parks, held twice each week for $1 \mathrm{hr}$ over 10 weeks. The sessions consisted of a 10-min warm-up, a continuous distance walk, time to practice specific outdoor walking skills (e.g., stopping suddenly, speeding up, walking on different surfaces), a second continuous distance walk, and a 10-min cooldown. Each week, the outdoor walking skills addressed two or more of the eight dimensions of outdoor mobility described by Patla and Shumway-Cook (1999). Target distances for the continuous walks ranged between 225 and $600 \mathrm{~m}$ for participants, and they were asked to try to complete the target distance for the walk if they were safely able to do so. For one walking session during Week 3 and one session during Week 9, participants wore ActiGraph GT3X+ monitors and Q-Starz BT-Q1000XT A-GPS devices on a belt over the right hip. The GT3X+ monitors were initialized using ActiLife6 software (version 6.13.3; ActiGraph LLC) to collect data at $100 \mathrm{~Hz}$. Qstarz QTravel software (Qstarz International Co., Ltd.) was used to set the GPS devices to log data every $1 \mathrm{~s}$. The supervisors of the walking sessions kept notes indicating when the activities started and ended and whether all participants engaged in all components of the session.

\section{Data Analysis}

Manual identification of walking bouts. GT3X+ activity monitor data were downloaded to obtain 5-s epoch files used to manually determine the start and end times of the continuous walking bouts. For this analysis, walking bouts $\geq 5$ min in duration were tracked, because they represent a level of walking necessary for sustained function in the community (e.g., to walk to a corner store or a bus stop; Clarke, Holdsworth, Ryan, \& Granat, 2013; Gardner et al., 2007). The start time of each bout was the time associated with the first instance of a step count $\geq 2$ (per 5-s epoch) that was followed by at least 60 epochs with step counts $\geq 2$ (allowing not more than 15 discontinuous epochs with step counts $<2$ ). When steps counts were $<2$ for $>8$ continuous epochs or $>15$ discontinuous epochs, the bout end time was recorded.
The low frequency extension (LFE) ActiGraph file was used to manually identify the bouts rather than the default file, because the total steps and, therefore, the step lengths recorded for the distances covered were more plausible with LFE. That is, the median step length (distance covered divided by number of steps) during the outdoor walks was $54 \mathrm{~cm}$ (interquartile range $[\mathrm{IQR}]=11 \mathrm{~cm}$ ) with the LFE analysis and $85 \mathrm{~cm}(\mathrm{IQR}=38 \mathrm{~cm})$ with the default analysis. Studies have demonstrated that step lengths typically range from 52 to $57 \mathrm{~cm}$ in older women and from 57 to $66 \mathrm{~cm}$ in older men (Hollman, McDade, \& Petersen, 2011; Shimada et al., 2010).

The global positioning system data were downloaded and the start and end times of each bout were marked as determined from the GT3X+ data. The Haversine formula was used to calculate the distance walked from latitude and longitude data (BlueMM, 2007). The accuracy of this technique was evaluated prior to the study over three walks ranging from 312 to $344 \mathrm{~m}$ in which distance was also measured with a 4-in. $(10.16-\mathrm{cm})$ diameter metric measuring wheel. Absolute percentage error for the GPS device distances ranged from $1 \%$ to $4 \%$. The average walking speed for each bout was determined as distance walked (in meters) divided by time (in seconds).

Identification of bouts using cadence thresholds and cpm threshold. The 5-s epoch files were reintegrated into 60-s files time synchronized with the GPS data using ActiLife6 software (version 6.13.3; ActiGraph LLC). A custom R program (https:// www.r-project.org/) was used to detect start and end times of walking bouts in which cadence was $\geq 30$ steps/min for a minimum of 5 min, allowing a maximum of 1 min below threshold (Figure 1). Analyses were also conducted to find start and end times of bouts using cadence thresholds $\geq 35, \geq 40, \geq 45$, and $\geq 50$ steps $/ \mathrm{min}$. ActiLife6 software (ActiGraph LLC) was used to detect 5-min lifestyle bouts (Axis 1 counts $>760 \mathrm{cpm}$ for a minimum of $5 \mathrm{~min}$, allowing a maximum of $1 \mathrm{~min}$ below threshold). All threshold analyses were conducted on both default and LFE files.

\section{Statistical Analysis}

Descriptive statistical analysis was conducted using SigmaPlot (version 11.0; Systat Software Inc., San Jose, CA). Sensitivity, specificity, Youden's index, positive predictive value (PPV), and

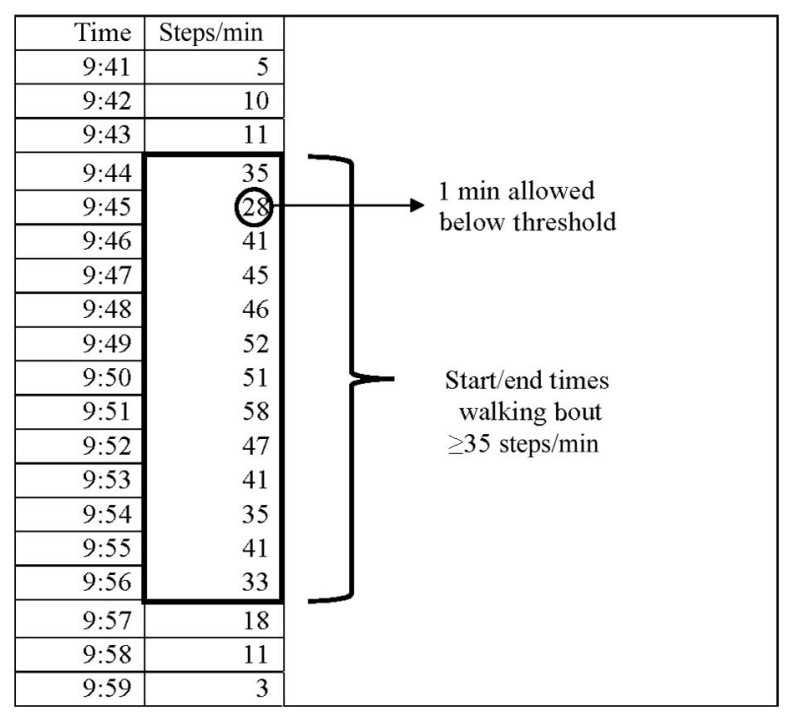

Figure 1 - Identification of start and end times of one walking bout using $\geq 35$ steps/min as the cadence threshold. 
negative predictive value results were estimated using SAS software (version 9.4; SAS Inc., Cary, NC). Sensitivity (Trevethan, 2017) of each analysis technique was estimated as the number of true positives (i.e., bouts that were identified manually in the 5-s epoch files that were also identified using the threshold analysis in the 60-s epoch files) divided by the sum of the number of true positives and the number of false negatives (i.e., bouts that were identified manually but were not identified using the threshold analysis). Specificity was estimated as the number of true negative walks (warm-up, cooldown, and intermittent walking skill practice times during the sessions when continuous walking did not occur) divided by the sum of true negative walks and false positive walks (which occurred when the threshold analysis detected a walk when none was identified manually). Youden's index (sensitivity + specificity-1), PPV, and negative predictive value were also estimated. We calculated exact $95 \%$ confidence intervals (CIs) assuming a binominal distribution. The theoretical distribution of Youden's index is not defined; therefore, 95\% CIs were not produced.

\section{Results}

Twenty-five older adults (19 women and six men) attended one or two supervised outdoor walking sessions. In those sessions, 15 participants completed a total of four continuous walks, three participants completed three walks, four participants completed two walks, and three participants completed one walk $(n=80$ walks, median duration $10.5 \mathrm{~min}, \mathrm{IQR}=4.8 \mathrm{~min}$; median outdoor walking speed $=0.70 \mathrm{~m} / \mathrm{s}, \mathrm{IQR}=0.20$ ). When walking outdoors, seven participants used a straight cane, four used a wheeled walker, and the remainder did not use a walking aid. Characteristics of participants and their walking capabilities are presented in Table 1.

Accuracy estimates for the different cadence and lifestyle cpm thresholds are presented in Table 2 . Sensitivity estimates (0.831.0) were higher when cadence thresholds were used to analyze LFE files compared with default files (range $=0.40-0.74$ ). Similarly, estimates of Youden's index were higher when analyses were conducted using the LFE files compared with the default files. Within the analyses conducted on LFE files, cadence thresholds of $\geq 30, \geq 35$, and $\geq 40$ steps/min (LFE files) demonstrated the greatest sensitivity in detecting walking bouts and produced high specificity estimates (0.95-0.97). While sensitivity and negative predictive value were the same for these three thresholds (all having values of $1.0), \geq 40 \mathrm{steps} / \mathrm{min}$ was associated with slightly greater estimates of specificity, Youden's index, and PPV. Using the lifestyle cpm threshold, sensitivity and specificity estimates for the LFE and default files were very similar. Sensitivity estimates associated with the lifestyle cpm threshold were substantially lower than those

\section{Table 1 Characteristics of Participants $(N=25)$}

\begin{tabular}{lc}
\hline Characteristic & Median (IQR) \\
\hline Age (years) & $77.0(10.5)$ \\
Height $(\mathrm{m})$ & $1.59(0.16)$ \\
Weight $(\mathrm{kg})$ & $70.5(38.8)$ \\
Body mass index $\left(\mathrm{kg} / \mathrm{m}^{2}\right)$ & $28.6(12.4)$ \\
10-m comfortable gait speed $(\mathrm{m} / \mathrm{s})$ & $0.97(0.25)$ \\
6MWT distance $(\mathrm{m})$ & $299.6(108.6)$ \\
6MWT speed $(\mathrm{m} / \mathrm{s})$ & $0.83(0.30)$ \\
\hline
\end{tabular}

Note. $\mathrm{IQR}=$ interquartile range; $6 \mathrm{MWT}=6$-min walk test. demonstrated using the $\geq 40$ steps/min (LFE) cadence threshold (0.86 vs. 1.0, respectively).

\section{Discussion}

The purpose of this study was to determine the optimal analysis technique for identifying sustained outdoor walking in mobilitylimited older adults, comparing different cadence thresholds and a lifestyle cpm threshold. Thresholds of $\geq 30, \geq 35$, and $\geq 40$ steps $/ \mathrm{min}$ (LFE files) all demonstrated high sensitivity (1.0, 95\% CI [0.95, 1.00]) in detecting known continuous outdoor walking that lasted at least $5 \mathrm{~min}$ in our sample. The threshold of $\geq 40 \mathrm{steps} / \mathrm{min}$ demonstrated slightly greater specificity and PPV compared with $\geq 30$ and $\geq 35$ steps/min. Cadence thresholds of $\geq 45$ and $\geq 50$ steps/min were less sensitive, as were all thresholds evaluated using the default ActiGraph files. The cpm threshold demonstrated moderate sensitivity and high specificity in detecting walking bouts, with no substantial difference when used on the LFE or default files. These findings have practical significance for measuring sustained walking from accelerometer data collected during free-living activity in mobility-limited older adults.

Cadence is a measure that is relatively easily understood by older adults and clinicians (Tudor-Locke, Craig, et al., 2011; Tudor-Locke \& Rowe, 2012). Median cadence values achieved during continuous walking in daily life have been shown to be associated with some mobility-related outcome measures such as gait speed and 400-m walk time (Urbanek et al., 2018). However, people demonstrate a wide range of cadence values throughout their daily routines (Tudor-Locke, Camhi, et al., 2011) and actually spend little time walking continuously for a number of minutes each day (Orendurff et al., 2008). For research purposes, it may be necessary to define a threshold that can be used to identify walking bouts that are relatively sustained, as opposed to sporadic stepping, in order to characterize free-living activity in older adults.

Tudor-Locke and Rowe (2012) suggested that cadences $<40$ steps/min represent incidental and sporadic movements, cadences of 40-59 steps/min indicate purposeful steps, and rhythmic slow walking occurs at $\geq 60$ steps/min. Our study is the first to measure cadences actually achieved by mobility-limited older adults during supervised outdoor walking sessions. During the continuous distance walks in each session, participants were encouraged to walk without stopping for a target distance, although they were permitted to take short breaks as needed. Our findings support Tudor-Locke's idea that cadences $\geq 40$ steps/min that are sustained for a number of minutes likely represent purposeful stepping and rhythmic slow walking for the majority of adults and some older adults. In addition, our results showed that for older adults who self-report outdoor mobility limitations, cadences as low as $\geq 30 \mathrm{steps} / \mathrm{min}$ also occur during periods of relatively sustained walking.

Although sensitivity in detecting sustained walking was high using thresholds of $\geq 30, \geq 35$, and $\geq 40$ steps/min, some episodes of sporadic walking (which occurred during warm-up/cooldown and walking skills activities) were incorrectly identified as continuous walking bouts at these lower thresholds. This is why specificity and PPV estimates were slightly lower for $\geq 30, \geq 35$, and $\geq 40$ steps $/ \mathrm{min}$ compared to the $\geq 45$ and $\geq 50$ steps/min thresholds. Researchers need to be aware that selecting a lower cadence for identifying sustained walking may result in mistakenly categorizing sporadic activities that occur repetitively as continuous walking bouts, whereas selecting a higher threshold may cause some continuous bouts of walking to go undetected (sensitivity decreases) if they do 
Table 2 Accuracy of Cadence and Lifestyle cpm Thresholds

\begin{tabular}{|c|c|c|c|c|c|c|}
\hline Analysis & $\geq 30$ steps $/ \mathrm{min}$ & $\geq 35$ steps/min & $\geq 40$ steps/min & $\geq 45$ steps $/ \mathrm{min}$ & $\geq 50$ steps $/ \mathrm{min}$ & $\geq 760 \mathrm{cpm}$ \\
\hline \multicolumn{7}{|l|}{ ActiLife LFE analysis } \\
\hline Sensitivity $(95 \%$ CI) & $1.00[0.95,1.00]$ & $1.00[0.95,1.00]$ & $1.00[0.95,1.00]$ & $0.89[0.80,0.95]$ & $0.83[0.72,0.90]$ & $0.86[0.77,0.93]$ \\
\hline Specificity $(95 \%$ CI) & $0.95[0.90,0.98]$ & $0.96[0.91,0.99]$ & $0.97[0.92,0.99]$ & $1.0[0.98,1.0]$ & $1.0[0.97,1.0]$ & $0.98[0.95,1.0]$ \\
\hline Youden's index & 0.95 & 0.96 & 0.97 & 0.89 & 0.83 & 0.85 \\
\hline PPV (95\% CI) & $0.93[0.85,0.97]$ & $0.94[0.87,0.98]$ & $0.95[0.88,0.99]$ & $1.0[0.95,1.0]$ & $1.0[0.95,1.0]$ & $0.97[0.90,1.0]$ \\
\hline NPV (95\% CI) & $1.0[0.97,1.0]$ & $1.0[0.97,1.0]$ & $1.0[0.97,1.0]$ & $0.93[0.88,0.97]$ & $0.90[0.84,0.95]$ & $0.92[0.86,0.96]$ \\
\hline \multicolumn{7}{|l|}{ ActiLife default analysis } \\
\hline Sensitivity $(95 \%$ CI) & $0.74[0.63,0.83]$ & $0.69[0.57,0.79]$ & $0.73[0.62,0.81]$ & $0.50[0.39,0.61]$ & $0.40[0.29,0.52]$ & $0.84[0.74,0.91]$ \\
\hline Specificity $(95 \%$ CI) & $0.99[0.96,1.0]$ & $1.0[0.97,1.0]$ & $1.0[0.97,1.0]$ & $1.0[0.97,1.0]$ & $1.0[0.97,1.0]$ & $1.0[0.97,1.0]$ \\
\hline Youden's index & 0.73 & 0.69 & 0.73 & 0.48 & 0.40 & 0.84 \\
\hline PPV (95\% CI) & $0.98[0.91,1.0]$ & $1.0[0.94,1.0]$ & $1.0[0.94,1.0]$ & $1.0[0.91,1.0]$ & $1.0[0.89,1.0]$ & $1.0[0.95,1.0]$ \\
\hline NPV $(95 \%$ CI $)$ & $0.86[0.79,0.91]$ & $0.84[0.77,0.89]$ & $0.85[0.79,0.91]$ & $0.76[0.69,0.83]$ & $0.73[0.66,0.79]$ & $0.91[0.85,0.95]$ \\
\hline
\end{tabular}

Note $. \mathrm{cpm}=$ counts per minute; $\mathrm{LFE}=$ low frequency extension; $\mathrm{CI}=$ confidence interval; $\mathrm{PPV}=$ positive predictive value; $\mathrm{NPV}=$ negative predictive value.

not meet the threshold for the required number of minutes. Our analyses demonstrated that $\geq 40$ steps/min represents the optimal threshold for detecting continuous outdoor walking in mobilitylimited older adults, because its sensitivity and specificity estimates were very high.

With the LFE files, the sensitivities associated with the lifestyle $(<760)$ cpm threshold $(0.86,95 \%$ CI $[0.77,0.93])$ and the cadence threshold of $\geq 45$ steps $/$ min $(0.89,95 \%$ CI $[0.80,0.95])$ were similar. This suggests that the intensity of activity during these walking bouts was similar to that achieved using a cpm threshold of $>760 \mathrm{cpm}$, which is thought to represent moderateintensity free-living activity in older adults.

There were some limitations associated with our study, in which data from 80 walks recorded in 25 participants were analyzed. Our sample consisted of older adults who self-reported having difficulty in walking outdoors. Gait speeds in our study were typical of those recorded in people with mobility limitations (Barclay et al., 2018; Peel, Kuys, \& Klein, 2013), but the sensitivity and specificity estimates associated with using cadence and cpm thresholds to detect walking may vary in older adults with different walking abilities and in different seasons. Similar analysis techniques could be used to determine appropriate thresholds for identifying sustained free-living walking in different samples. The 5-s epoch step data were analyzed to manually identify the start and end times of the walking bouts. Using this technique, it is possible that sporadic activity (e.g., in the last minutes of the warm-up or the first minutes of the cooldown) may have been mistakenly counted as part of the continuous walking time. Steps were not directly observed during the supervised walking sessions. While ActiLife's LFE filter has been shown to be accurate for use in individuals with gait speeds $<1.0 \mathrm{~m} / \mathrm{s}$ (Bertapelli et al., 2019; Korpan, Schafer, Wilson, \& Webber, 2015; Webber \& St. John, 2016), the absolute percentage error may be as high as $7 \%$ in individuals who walk slowly and use a walking aid (Korpan et al., 2015).

It is important to recognize that steps and steps per min represent variables that are inherently understood by older adults and clinicians (Tudor-Locke, Craig, et al., 2011; Tudor-Locke \& Rowe, 2012). Therefore, using steps and cadence as outcomes may make findings of physical activity research more relevant to a broader audience of individuals from different disciplines. The threshold of $\geq 40$ steps/min (LFE files) demonstrated the highest sensitivity and specificity estimates for detecting continuous walking bouts in our sample of community ambulators who reported difficulties with outdoor mobility. Future studies should continue to evaluate typical cadence levels achieved during sustained walking in people of different ages and abilities during freeliving activity.

\section{Acknowledgment}

This study was funded by a project scheme grant from the Canadian Institutes of Health Research, grant number 376439.

\section{References}

ActiGraph. (2008). ActiGraph white paper: What is a count? Retrieved from https://www.actigraphcorp.com/white-papers/

Barclay, R., Webber, S., Ripat, J., Grant, T., Jones, C.A., Lix, L.M., ... Salbach, N.M. (2018). Safety and feasibility of an interactive workshop and facilitated outdoor walking group compared to a workshop alone in increasing outdoor walking activity among older adults: A pilot randomized controlled trial. Pilot and Feasibility Studies, 4, 179. doi:10.1186\%2Fs40814-018-0367-4

Bertapelli, F., Curtis, J.S., Carlson, B., Johnson, M., Abadie, B., \& Agiovlasitis, S. (2019). Step-counting accuracy of activity monitors in persons with Down syndrome. Journal of Intellectual Disability Research, 63(1), 21-30. PubMed ID: 30239068 doi:10.1111/jir.12550

BlueMM. (2007, January 6). Excel formula to calculate distance between 2 latitude, longitude (lag/lon) points (GPS positions). Retrieved from http:// bluemm.blogspot.com/2007/01/excel-formula-to-calculate-distance.html

Clarke, C.L., Holdsworth, R.J., Ryan, C.G., \& Granat, M.H. (2013). Freeliving physical activity as a novel outcome measure in patients with intermittent claudication. European Journal of Vascular and Endovascular Surgery, 45(2), 162-167. PubMed ID: 23265683 doi:10. 1016/j.ejvs.2012.11.027

Gardner, A.W., Montgomery, P.S., Scott, K.J., Afaq, A., \& Blevins, S.M. (2007). Patterns of ambulatory activity in subjects with and without intermittent claudication. Journal of Vascular Surgery, 46(6), 12081214. PubMed ID: 17919876 doi:10.1016/j.jvs.2007.07.038

Gennuso, K.P., Gangnon, R.E., Matthews, C.E., Thraen-Borowski, K.M., \& Colbert, L.H. (2013). Sedentary behavior, physical activity, and markers of health in older adults. Medicine \& Science in Sports \& 
Exercise, 45(8), 1493-1500. PubMed ID: 23475142 doi:10.1249/ MSS.0b013e318288a1e5

Giannouli, E., Bock, O., Mellone, S., \& Zijlstra, W. (2016). Mobility in old age: Capacity is not performance. BioMed Research International, 2016, 3261567. doi:10.1155/2016/3261567

Granat, M., Clarke, C., Holdsworth, R., Stansfield, B., \& Dall, P. (2015). Quantifying the cadence of free-living walking using event-based analysis. Gait \& Posture, 42(1), 85-90. PubMed ID: 25953505 doi:10.1016/j.gaitpost.2015.04.012

Hall, K.S., Howe, C.A., Rana, S.R., Martin, C.L., \& Morey, M.C. (2013). METs and accelerometry of walking in older adults: Standard versus measured energy cost. Medicine \& Science in Sports \& Exercise, 45(3), 574-582. PubMed ID: 23059862 doi:10.1249/MSS.0b013e318276c73c

Harada, N.D., Chiu, V., \& Stewart, A.L. (1999). Mobility-related function in older adults: Assessment with a 6-minute walk test. Archives of Physical Medicine and Rehabilitation, 80(7), 837-841. PubMed ID: 10414771 doi:10.1016/S0003-9993(99)90236-8

Hollman, J.H., McDade, E.M., \& Petersen, R.C. (2011). Normative spatiotemporal gait parameters in older adults. Gait \& Posture, 34(1), 111-118. PubMed ID: 21531139 doi:10.1016/j.gaitpost. 2011.03 .024

Korpan, S.M., Schafer, J.L., Wilson, K.C., \& Webber, S.C. (2015). Effect of ActiGraph GT3X+ position and algorithm choice on step count accuracy in older adults. Journal of Aging and Physical Activity, 23(3), 377-382. PubMed ID: 25102469 doi:10.1123/japa.2014-0033

Matthew, C.E. (2005). Calibration of accelerometer output for adults. Medicine \& Science in Sports \& Exercise, 37(11, Suppl.), S512-S522. PubMed ID: 16294114 doi:10.1249/01.mss.0000185659.11982.3d

Orendurff, M.S., Schoen, J.A., Bernatz, G.C., Segal, A.D., \& Klute, G.K. (2008). How humans walk: Bout duration, steps per bout, and rest duration. Journal of Rehabilitation, Research and Development, 45(7), 1077-1089. doi:10.1682/JRRD.2007.11.0197

Paraschiv-Ionescu, A., Bula, C.J., Major, K., Lenoble-Hoskovec, C., Krief, H., El-Moufawad, C., \& Aminian, K. (2018). Concern about falling and complexity of free-living physical activity patterns in wellfunctioning older adults. Gerontology, 64(6), 603-611. PubMed ID: 29972821 doi:10.1159/000490310

Patla, A.E., \& Shumway-Cook, A. (1999). Dimensions of mobility: Defining the complexity and difficulty associated with community mobility. Journal of Aging and Physical Activity, 7(1), 7-19. doi:10. 1123/japa.7.1.7

Peel, N.M., Kuys, S.S., \& Klein, K. (2013). Gait speed as a measure in geriatric assessment in clinical settings: A systematic review. Journals of Gerontology, Series A: Biological Sciences \& Medical Sciences, 68(1), 39-46. PubMed ID: 22923430 doi:10.1093/ gerona/gls 174

Rydwik, E., Bergland, A., Forsen, L., \& Frandin, K. (2012). Investigation into the reliability and validity of the measurement of elderly people's clinical walking speed: A systematic review. Physiotherapy Theory and Practice, 28(3), 238-256. PubMed ID: 21929322 doi:10.3109/ 09593985.2011 .601804

Salbach, N.M., Barclay, R., Webber, S.C., Jones, C.A., Mayo, N.E., Lix, L.M., ... Chilibeck, P.D. (2019). A theory-based, task-oriented, outdoor walking programme for older adults with difficulty walking outdoors: Protocol for the Getting Older Adults Outdoors (GO-OUT) randomised controlled trial. BMJ Open, 9, 3029393. doi:10.1136/ bmjopen-2019-029393

Schrack, J.A., Cooper, R., Koster, A., Shiroma, E.J., Murabito, J.M., Rejeski, W.J., ... Harris, T.B. (2016). Assessing daily physical activity in older adults: Unraveling the complexity of monitors, measures, and methods. Journals of Gerontology, Series A: Biological Sciences \& Medical Sciences, 71(8), 1039-1048. PubMed ID: 26957472 doi:10.1093/gerona/glw026

Shimada, H., Kim, H., Yoshida, H., Suzukawa, M., Makizako, H., Yoshida, Y., ... Suzuki, T. (2010). Relationship between ageassociated changes of gait and falls and life-space in elderly people. Journal of Physical Therapy Science, 22(4), 419-424. doi:10.1589/ jpts.22.419

Trevethan, R. (2017). Sensitivity, specificity, and predictive values: Foundations, pliabilities, and pitfalls in research and practice. Front Public Health, 5, 307. PubMed ID: 29209603 doi:10.3389/fpubh. 2017.00307

Tryon, W.W., \& Williams, R. (1996). Fully proportional actigraphy: A new instrument. Behavior Research Methods, Instruments, and Computers, 28(3), 392-403. doi:10.3758/BF03200519

Tudor-Locke, C., Barreira, T.V., Brouillette, R.M., Foil, H.C., \& Keller, J.N. (2013). Preliminary comparison of clinical and free-living measures of stepping cadence in older adults. Journal of Aging and Physical Activity, 10(8), 1175-1180. doi:10.1123/jpah.10.8.1175

Tudor-Locke, C., Camhi, S.M., Leonardi, C., Johnson, W.D., Katzmarzyk, P.T., Earnest, C.P., \& Church, T.S. (2011). Patterns of adult stepping cadence in the 2005-2006 NHANES. Preventive Medicine, 53(3), 178-181. PubMed ID: 21708187 doi:10.1016/j.ypmed. 2011.06.004

Tudor-Locke, C., Craig, C.L., Aoyagi, Y., Bell, R.C., Croteau, K.A., De Bourdeaudhuij, I., ... Blair, S.N. (2011). How many steps/day are enough? For older adults and special populations. International Journal of Behavioral Nutrition and Physical Activity, 8, 80. PubMed ID: 21798044 doi:10.1186/1479-5868-8-80

Tudor-Locke, C., \& Rowe, D.A. (2012). Using cadence to study free-living ambulatory behaviour. Sports Medicine, 42(5), 381-398. PubMed ID: 22462794 doi:10.2165/11599170-0000 00000-00000

Urbanek, J.K., Zipunnikov, V., Harris, T., Crainiceanu, C., Harezlak, J., \& Glynn, N.W. (2018). Validation of gait characteristics extracted from raw accelerometry during walking against measures of physical function, mobility, fatigability, and fitness. Journals of Gerontology, Series A: Biological Sciences \& Medical Sciences, 73(5), 676-681. PubMed ID: 28958000 doi:10.1093/gerona/glx 174

Valenti, G., Bonomi, A.G., \& Westerterp, K.R. (2016). Walking as a contributor to physical activity in healthy older adults: 2 week longitudinal study using accelerometry and the doubly labeled water method. JMIR mHealth uHealth, 4(2), e56. PubMed ID: 27268471 doi:10.2196/mhealth.5445

Webber, S.C., \& St. John, P.D. (2016). Comparison of ActiGraph GT3X+ and StepWatch step count accuracy in geriatric rehabilitation patients. Journal of Aging and Physical Activity, 24(3), 451-458. PubMed ID: 26751505 doi:10.1123/japa.2015-0234 